\title{
Sozialpädagogisches Wissen vermitteln
}

\section{Ebenen und Zielgruppen der Vermittlung einer sozialpädagogischen Didaktik für berufsbil- dende Schulen}

Im Spannungsfeld zwischen dem steigenden Fachkräftebedarf in den Handlungsfeldern der Sozialen Arbeit, dem Fachkräftegebot einschließlich entsprechender Diskussionen zur Professionalisierung des pädagogischen Personals sowie den unterschiedlichen Orten und Niveaustufen der möglichen Qualifikationen sind berufsbildende Schulen ein zentrales Element der Qualifikationslandschaft. Dies wirft die Frage danach auf, wo sich insbesondere die Erzieher_innenausbildung innerhalb dieses Diskurses positioniert und was bei der Vermittlung sozialpädagogischer Kompetenzen im Übergang der verschiedenen Bildungsorte zu beachten ist.

I $\mathrm{n}$ den sozialpädagogischen Theoriediskussionen lässt sich, mit wenigen Ausnahmen, eine gewisse Didaktik-Abstinenz ausmachen. Dabei gilt für die Sozialpädagogik und ihre Didaktik als Fach- bzw. Berufsfelddidaktik ähnliches wie für die Sozialpädagogik als Fachwissenschaft. Geht es bei der Sozialpädagogik vornehmlich darum, das Disziplinäre als „fixiertes Gedankengebäude“ zu skizzieren, geht es mit Blick auf

Mischa Engelbracht'1,2
$\begin{aligned} & \text { 'Universität Erfurt, Erfurt, Deutschland } \\ & \text { 'Bergische Universität Wuppertal, Wuppertal, Deutschland } \\ & \text { *1987, Studium der Sozialen Arbeit an der Universität Kassel, } \\ & \text { Studium der Erziehungswissenschaft an der WWU Münster, } \\ & \text { Promotion an der Technischen Universität Dresden, Akademi- } \\ & \text { scher Rat an der Universität Erfurt. Zurzeit Vertretungsprofessor } \\ & \text { für Sozialpädagogik und ihre Didaktik an der Bergischen } \\ & \text { Universität Wuppertal. } \\ & \text { mischa.engelbracht@uni-erfurt.de }\end{aligned}$

Zusammenfassung Wenn über die Vermittlung sozialpädagogischen Wissens nachgedacht wird, stellt insbesondere die Breite des Feldes der sozialpädagogischen Qualifikationsangebote eine besondere Herausforderung dar. Neben verschiedenen Orten finden sich unterschiedliche Niveaustufen der Qualifikation. Ein Ort sind dabei die Fachschulen für Sozialpädagogik, an denen auf dem Niveau der Kinderpfleger_innen/ Sozialassistent_innen (DQR 4) und Erzieher_innen (DQR 6) ausgebildet wird. Dem Prinzip der doppelten Vermittlungspraxis folgend, gilt es für Überlegungen zur Sozialpädagogik und ihrer Didaktik entsprechend die Orte, Qualifikationsstufen und auch den Transfer des Wissens zwischen den Orten und Niveaustufen zu berücksichtigen.

Schlüsselwörter Sozialpädagogik und ihre Didaktik, (Doppelte) Vermittlungspraxis, Didaktische Prinzipien, Berufsbildende Schulen die Didaktik wohl bislang eher praktisch darum, etwa in den Fachschulen für Sozialpädagogik, ihre „gesellschaftliche Realität“ (vgl. Hamburger 2008, S. 11) zu beschreiben.

Eine besondere Herausforderung stellt bei der Vermittlung sozialpädagogischen Wissens dabei die Breite des Feldes der sozialpädagogischen Qualifikationsangebote dar, welches - historisch gewachsen - durchaus als komplex bezeichnet werden kann. Diese Komplexität wird im Kontext der Vermittlungspraxis insbesondere dann relevant, wenn es um die Relationierung der verschiedenen Orte der Qualifikation (Fachschule, Berufsakademie, Hochschulen für angewandte Wissenschaft, Universitäten) und auch mitunter gleichwertigen, aber nicht gleichartigen Niveaustufen (Niveau 4: Kinderpflege, Sozialassistenz; Niveau 6: Erzieher_innen, Bachelor-Absolvent_innen; Niveau 7: Master-Absolvent_innen) geht.

Zumindest im Regelfall ermöglicht die jeweilige Qualifikation, unabhängig von Ort und Niveau, eine spätere sozialpädagogische Tätigkeit, womit die Frage nach dem Lernen und Lehren sozialpädagogischer Kompetenzen in den Fokus rückt. Der vorliegende Beitrag fokussiert die unterschiedlichen Ebenen und Zielgruppen der sozialpädagogischen Vermittlungspraxis unter Fragen nach Zusammenhängen von Statusübergängen und didaktischen Überlegungen für das Lehramt Sozialpädagogik in berufsbildenden Schulen. Die Auseinandersetzung mit entsprechenden didaktischen Diskussionen ist in ihrer Bedeutung kaum zu überschätzen, immerhin stellt die Berufsgruppe der Erzieher_innen nach wie vor den größten Teil der pädagogischen Fachkräfte in Deutschland (vgl. Autor" innengruppe Fachkräftebarometer 2019, S. 83 ff). 


\section{Durchblick: Neuformatierung d. sozialpädagog. Qualifizierungslandschaft}

\section{Sozialpädagogik und Didaktik}

Die Sozialpädagogik hat viele Gesichter und ,ist nicht nur eine erziehungs- und damit sozialwissenschaftliche Disziplin im allgemeinen Sinne, sondern gleichzeitig auch eine Theorie besonderer Praxisinstitutionen (...). Als erziehungswissenschaftliche Disziplin beschäftigt sich die Sozialpädagogik mit jenen sozialstrukturell und institutionell bedingten Konflikten, welche im Verlauf der Sozialisation von Kindern und Jugendlichen auftreten: Konflikte zwischen subjektiven Antrieben und Vermögen sowie gesellschaftlichen und institutionellen Anforderungen, wie sie in Familie, Schule, Arbeitswelt und Gemeinwesen vermittelt sind. Sie versucht, diese Konflikte aufzuklären, ihre Folgeprobleme zu prognostizieren und in diesem Kontext Grundlagen für erzieherische Hilfen zu entwickeln “ (Böhnisch 1979, S. 22). Die Sozialpädagogik orientiert sich in ihren Grundfragen also daran, wie sich ein „gutes Leben“ oder „gelingende Sozialisation“ über Erziehung, Bildung, Hilfe oder auch Förderung ermöglichen oder unterstützen lässt. Die Relationierung von Sozialpädagogik zu ihren didaktischen Fragen ist in unterschiedlichen Zugängen, Konzepten oder theoretischen Verortungen allerdings oft unklar. Dies ist zunächst wenig verwunderlich, immerhin hat die Sozialpädagogik sich mit einer vergleichsweise deutlichen Abgrenzung zu schulischen Fragen und Themen etabliert (Gängler 1995).

In Folge professionalisierungstheoretischer Überlegungen rückt das Ziel der Befähigung zu einem ausgewiesenen fachlichen, eben professionellen Handeln und daran anschließender fachdidaktischer Themen stärker in den Fokus, auch der fachwissenschaftlichen Öffentlichkeit. Was zu einer stärkeren Auseinandersetzung, in Form einer Annäherung, zwischen sozialpädagogischen und didaktischen Diskursen führt (Liebig 2020; Göddertz 2021 in diesem Schwerpunkt). Wenn es auch historisch gewachsen deutliche Differenzen zwischen beiden erziehungswissenschaftlichen Teildisziplinen gibt, scheinen sich doch beispielsweise mit Wolfgang Klafkis „Neue Studien zur Bildungstheorie und Didaktik“ (1996) und dem lebensweltorientierten Bewältigungsansatz (Thiersch und Böhnisch 2014; Thole 2012, S. 42) durchaus weitere Verknüpfungen zwischen didaktischen und sozialpädagogischen Argumentationen und normativen Zielsetzungen herzustellen. So führt Klafki aus, dass die kritisch-konstruktive Didaktik als eine „übergreifende Bezeichnung für erziehungswissenschaftliche Forschung, Theorie und Konzeptbildung im Hinblick auf alle Formen intentionaler (zielgerichteter), systematisch vorbedachter ,Lehre“ (im weitesten Sinne von reflektierter Lern-Hilfe) und auf das im Zusammen- hang mit solcher ,Lehre‘ sich vollziehende Lernen zu bezeichnen" (Klafki 1996, S. 91) ist.

Ziel einer kritischen Erziehungswissenschaft und ihrer Didaktik ist weiter die Befähigung zur Selbstbestimmung, Mitbestimmung und Solidarität in allen Lebensdimensionen (Klafki 1996, S. 89), was sich mit der in der Sozialpädagogik viel zitierten Forderung der „Erziehung zur Mündigkeit“ (Adorno 1971), dem Verständnis von allgemeiner Bildung bei Hans Thiersch (1979) oder Bildung bei Heinz Sünker (2009) verknüpfen ließe. Dass Lernprozesse, als Aneignungsprozesse, sich nicht auf Orte wie die Schule oder Lebensalter wie die Kindheit beschränken lassen, sondern über die gesamte Lebensspanne an allen Orten stattfinden können, ist unstrittig. Im Kontext der Didaktik sind dabei insbesondere intendierte, also geplante, Lernprozesse relevant (Gängler und Liebig 2017). Im Kontext der Sozialpädagogik besonders solche, die auf die konkreten Aufgaben des Alltags abzielen (Thiersch 1979). Diese sollten sich auf die Alltagserfahrungen und Alltagssituationen der Lernenden beziehen, über indirekte Arrangements (didaktische Lernsituationen) angeregt werden und sich auf gemeinsame Erfahrungen, dem Miteinander beziehen (Thiersch 1979). Eine Didaktik der Sozialpädagogik stellt somit die Frage danach, wie über intentional vorbedachter Lernsituationen junge Menschen in ihrem Alltag lernen und dabei begleitet werden können, an Gesellschaft im Sinne einer mündigen Selbstbestimmung zu partizipieren.

Lehrende, pädagogische Fachkräfte, aber auch Studierende, Schüler_innen an berufsorientierten Schulen oder Kinder und Jugendliche bewegen sich als Subjekte im pädagogischen Raum und sind somit auch in ihrer Körperlichkeit oder Selbstpositionierung Teil des pädagogischen wie didaktischen Settings und Interaktionsprozesses. Alle Akteur_innen sind Co-Produzent_innen des Alltags und gestalten so gemeinsam ihre Lernarrangements. Diese Prämissen gelten mit Blick auf die integrale Persönlichkeitsentwicklung (Liebig und Gängler 2018) sowohl für die angehenden Erzieher_innen, aber auch für die angehenden Lehrer_innen. Wenn es also um die Qualifikation der pädagogischen Fachkräfte geht, scheint das Bildungs-, Lern- und auch Lehrverständnis durchaus mit Teilen der didaktischen Diskussionen übereinzustimmen. Wobei es eine deutliche, strukturelle Differenz zwischen der sozialpädagogischen Perspektive auf Didaktik zu etwa allgemeinen didaktischen Überlegungen gibt.

\section{Die doppelte Vermittlungspraxis als didaktischen Prinzip}

„[Didaktische Prinzipien] werden zur Begründung didaktischen Handelns herangezogen und können als 
handlungsleitende Grundsätze verstanden werden, die eine Orientierungsfunktion bieten und zugleich Handlungs- und Entscheidungsräume eröffnen. Daher stehen didaktische Prinzipien in Verknüpfung mit empirischen Befunden, Theorien und normativen Bezügen und stellen somit eine Brückenfunktion von didaktischer Theorie und didaktischer Praxis da" (Göddertz und Karber 2019, S. 67). Wenn die didaktischen Prinzipien in der Sozialpädagogik nach wie vor auch unzureichend theoretisch fixiert scheinen, so sind sie dennoch Bestandteil der entsprechenden Lehrpläne (KMK 2019). Für die Sozialpädagogik gilt entsprechend der Lehrpläne bislang die „integrale Persönlichkeitsentwicklung“, die „Theorie-Praxis-Relation“ und die „doppelte Vermittlungspraxis“ als Prinzipien (KMK 2019). Der vorliegende Beitrag fokussiert dabei das Prinzip der „doppelten Vermittlungspraxis“ bzw. des doppelten pädagogischen Bezugs im Lehramt Sozialpädagogik, in welchen auch die oben angeführten strukturellen Differenzen deutlich werden. Während in den allgemein didaktischen Überlegungen und Modellen immer die Schüler_innen als Lernende sich Wissen zu einem Unterrichtsfach (etwa Mathematik) aneignen, muss in den sozialpädagogischen didaktischen Modellen, die für die berufsbildenden Schulen entwickelt werden, immer die Vermittlung an die anschließende Zielgruppe mitgedacht werden. Die doppelte Vermittlungspraxis in der Sozialpädagogik bezieht sich also als didaktischer Ort sowohl auf die Schule also auch auf Praxisfelder. Es gilt, damit die beiden Orte der Vermittlung, berufsbildende Schule und Praxisfelder (beispielsweise Kindertageseinrichtungen, Einrichtungen der Hilfen zur Erziehung) und ihre jeweilige Zielgruppe als Adressat_innen sozialpädagogischer Lehr- und Lernangebote oder Nutzer_innen didaktischer Orte im Blick zu behalten. In Bezug auf die Lehramtsausbildung bedeutet dies, „dass es keinesfalls ausreicht, jemanden beizubringen, wie er selbst sozialpädagogisch zu handeln hat, sondern es muss ein weiterer Schritt bedacht werden, der sich mit der Frage umreißen lässt: Wie kann Studierenden vermittelt werden, selbst Sozialpädagogik so zu lehren, dass diese später an den jeweiligen Schulen ihren Schülerinnen und Schülern kompetentes sozialpädagogisches Handeln vermitteln können?" (Gängler 2010, S. 369). Eine sozialpädagogische Didaktik muss sich demzufolge immer auf verschiedene Orte und Zielgruppen beziehen.

Die von Hans Gängler (2010) aufgeworfene Frage kann allerdings noch einmal erweitert werden. Insbesondere wenn eine Verschiebung der Perspektive von den Orten der Vermittlung auf die theoretischen Wissensbestände vorgenommen wird, die auf der einen Seite die gesamte Breite der Theoriediskussion der Qualifika- tionslandschaft in den Blick nimmt und auf der anderen Seite auch die Besonderheit der institutionellen Übergänge berücksichtigt. So ist zu fragen,

- wie Kinder und Jugendliche als Co-Produzent_innen mit ihren Erzieher_innen alltägliche Lernarrangements so gestalten, dass diese professionelles pädagogisches Handeln und wissenschaftliche sozialpädagogische Zielsetzungen erkennen lassen,

- welches die Erzieher_innen als vormalige Schüler_innen sich als Co-Produzent_innen mit ihren Lehrer_innen in Berufskollegs haben erarbeiten können oder

- welches aus dem Transferprozess der Lehrer_innen als vormalige Studierende in der Universität hervorgeht und welches diese sich mit Dozierenden als Lernbegleiter haben aneignen können.

Eine Didaktik für das Lehramt Sozialpädagogik an berufsbildenden Schulen muss also Antworten auf die folgenden Fragen mitdenken:

1. Was benötigen die Kinder und Jugendlichen und ihre Familien als Nutzer_innen im Sinne von CoProduzent_innen der Angebote und wie wird das Erlernen ermöglicht?

2. Wie kann dies in der Interaktion von Erzieher_innen und Nutzer_innen gelingen?

3. Wie gestaltet sich der Statusübergang von Schüler_ in zu Erzieher_in?

4. Was brauchen die Schüler_innen als Nutzer_innen der Angebote und welche Bedingungen für Lernprozesse sind zu schaffen?

5. Wie kann dies in der Interaktion von Lehrer_innen und Schüler_innen gelingen?

6. Wie gestaltet sich der Rollenübergang von Studierende zu Lehrer_innen gestaltet?

7. Was brauchen die Studierenden als Nutzer*innen der Angebote und wie wird hier das Erlernen ermöglicht?

8. Wie kann dies in der Interaktion von Dozierenden und Studierenden gelingen?

9. Was brauchen die Dozierenden?

Das Verhältnis von Inhalten, Lehrenden und Lernenden sowie ihre Übergänge in Lehrende kann in Form von didaktischen Dreiecken dargestellt werden (vgl. Abb. 1). In diesem Fall als Symbol für den jeweiligen Ort der doppelten Vermittlungspraxis der Hochschule mit Fokus auf Berufskollegs und Praxisfeld. Auch wenn die sozialpädagogische Berufsfelddidaktik auf Lernen und Lehren in Fach- und Berufsschulen abzielt, so darf doch der Fokus auf die beiden Zielgruppen, den angehenden Erzieher_innen, aber auch insbesondere der Kinder und Jugendlichen, nicht verloren gehen. Immer- 


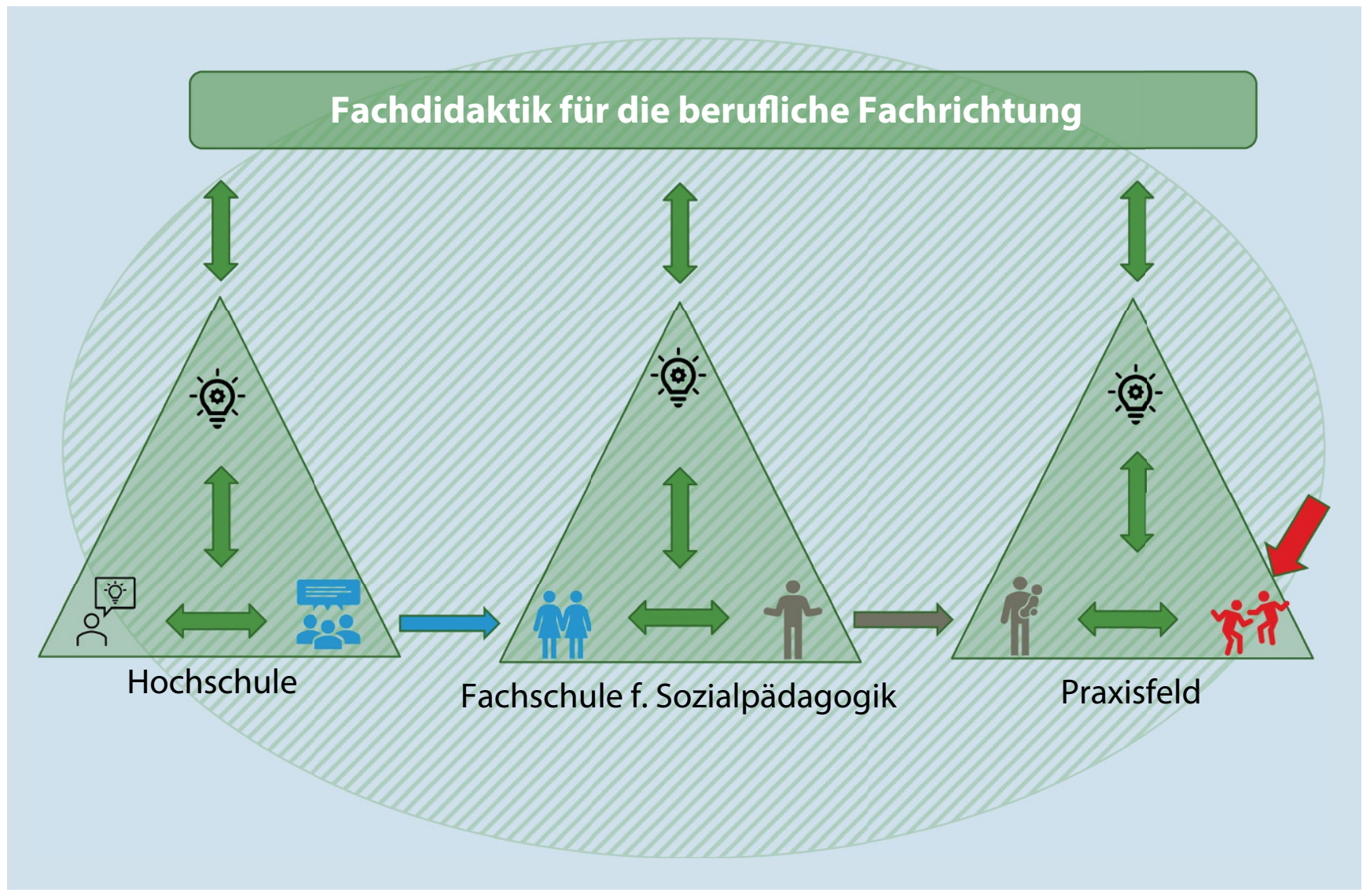

Abb. 1 Ebenen und Zielgruppen für die berufliche Fachrichtung Sozialpädagogik

hin folgt weder das Lehramtsstudium noch die Ausbildung zum/zur Erzieher_in einem Selbstzweck. Die Gesamtheit der pädagogischen Prozesse vollzieht sich dabei vor dem Hintergrund der gesellschaftlichen Bedingungen (vgl. ovaler Hintergrund in Abb. 1).

\section{Perspektiven}

Um der Zielsetzung, professionelles sozialpädagogisches Handeln zu ermöglichen, zu entsprechen, kommt der Qualifikation von Fachkräften eine besondere Bedeutung zu. Dass sich auch die Qualifikation der sozialpädagogischen Lehramtsstudierenden dem Spannungsverhältnis von Allgemeinbildung und Ausbildung ausgesetzt sieht und die Verschulung der Studiengänge einem stärkeren Ausbildungscharakter folgt, dürfte wenig strittig sein (vgl. Gruschka 2011, S. 66 ff.). Dass eine entsprechende Erwartungshaltung auch zunächst von Studierenden (und später auch Schüler_innen) an die jeweiligen Orte der Qualifikation herangetragen wird, ist wenig verwunderlich. Problematisch wird es, wenn die Frage „nach praktikablen Handlungsmustern auf technische Aspekte verkürzt“ und als reines „,Rezeptwissen', das klare Regeln vorgibt, die man im konkreten Fall nur benutzen muss, um das jeweilige Problem zu lösen " heruntergebrochen wird (Galuske 2013, S. 19). Wenn in einer sozialpädagogischen Didaktik der Fokus vom genuinen wissenschaftlichen Verstehen auf anwendbare Handlungsmuster verkürzt würde, hätte dies in der Folge einen Ausschluss der übrigen oben ausgeführten Ebenen zur Folge. Die Perspektive auf die Kinder- und Jugendlichen als zentrale Zielgruppe würde verloren gehen. Methodisierungen im Sinne von Rezeptwissen zielt auf die Reproduktion spezifischer Handlungen in spezifischen Kontexten ab und kann in keinem Fall eine zweite Ebene von Zielgruppen fokussieren.

Die Sozialpädagogik und ihre Didaktik müssen, so argumentiert, auf das Ziel des Verstehens und des Überzeugens setzen. Studierende respektive Lehrer_innen ebenso wie Schüler_innen und Erzieher_innen müssen eine Orientierung an Selbstbestimmung, gesellschaftlicher Partizipation und demokratischen Prinzipien ihrem pädagogischen Handeln zu Grunde legen, wie es etwa im Setting der Heimerziehung am Beispiel der Wahlen zu Gruppenräten deutlich wird (Engelbracht und Leissenberger 2019): Das Wissen, etwa wie methodisch Wahlen zu einem Gruppenrat abgehalten werden, wird so zweitrangig, ebenso wie das Exerzieren der konzeptionierten Anweisung, einen Rat wählen lassen zu müs- 
sen. Ohne grundlegender Überzeugung, welche von der Universität, über berufsbildende Schulen bis in die Praxisfelder transferiert werden muss, erfüllt das reine formal korrekte Abhalten einer Wahl nicht die Ziele und damit didaktischen Prinzipien der Sozialpädagogik. Umso wichtiger scheint es für die anstehenden Didaktik-Diskussionen - einschließlich des Wissenstransfers zwischen den verschiedenen Lernorten -, das Verhältnis von methodisiertem pädagogischen Wissen und einer verstehenden pädagogischen Überzeugung präzise weiter auszuarbeiten. Eine Überzeugung, die auf didaktischen Prinzipien beruht und aus der sich deutlich ableiten lässt, dass Studierende, Schüler_innen oder auch Kinder und Jugendliche keine Konsument_innen vorproduzierter Inhalte sein sollten. Pädagogisch gesehen geht es nicht um die einfache Reproduktion von vorstrukturieren Handlungsabläufen, sondern vielmehr um die Co-Produktion partizipativer Lernarrangements im sozialpädagogischen Alltag, welche allen Akteur_innen Lernprozesse - auch zur weiteren Persönlichkeitsbildung und -entwicklung - ermöglichen.

Eingegangen. 20. Juni 2021

Angenommen. 16. Juli 2021

Funding. Open Access funding enabled and organized by Projekt DEAL.

Open Access. Dieser Artikel wird unter der Creative Commons Namensnennung 4.0 International Lizenz veröffentlicht, welche die Nutzung, Vervielfältigung, Bearbeitung, Verbreitung und Wiedergabe in jeglichem Medium und Format erlaubt, sofern Sie den/die ursprünglichen Autor(en) und die Quelle ordnungsgemäß nennen, einen Link zur Creative Commons Lizenz beifügen und angeben, ob Änderungen vorgenommen wurden.

Die in diesem Artikel enthaltenen Bilder und sonstiges Drittmaterial unterliegen ebenfalls der genannten Creative Commons Lizenz, sofern sich aus der Abbildungslegende nichts anderes ergibt. Sofern das betreffende Material nicht unter der genannten Creative Commons Lizenz steht und die betreffende Handlung nicht nach gesetzlichen Vorschriften erlaubt ist, ist für die oben aufgeführten Weiterverwendungen des Materials die Einwilligung des jeweiligen Rechteinhabers einzuholen.

Weitere Details zur Lizenz entnehmen Sie bitte der Lizenzinformation auf http://creativecommons.org/licenses/by/4.0/deed.de.

\section{Literatur}

Adorno, T. W. (1971). Erziehung zur Mündigkeit. In G. Kadelbach (Hrsg.), Erziehung zur Mündigkeit. Vorträge und Gespräche mit Hellmut Becker 1959-1969 (S. 133-147). Frankfurt a.M.: Suhrkamp.

Autor*innengruppe Fachkräftebarometer (2019). Fachkräftebarometer Frühe Bildung 2019. Weiterbildungsinitiative Frühpädagogische Fachkräfte (WiFF). München: DJI.
Böhnisch, L. (1979). „Sozialpädagogik“ hat viele Gesichter. Betrifft: Erziehung: b:e; das aktuelle pädagogische Magazin; Forum für Bildungspolitik und Erziehungswissenschaft, 9, 22-24.

Engelbracht, M., \& Leissenberger, F. (2019). Teilhabe trotz Sozialpädagogik? Partizipation und freiheitsentziehende Maßnahmen. In Kommission Sozialpädagogik (Hrsg.), Teilhabe durch *in"trotz Sozialpädagogik (S. 132145). Weinheim \& Basel: Beltz Juventa.

Galuske, M. (2013). Methoden der Sozialen Arbeit. Eine Einführung. Weinheim \& Basel: Beltz Juventa.

Gängler, H. (1995). Sozialpädagogik als Wissenschaft. Studien zur Wissenschaftsgeschichte der Sozialpädagogik. Manuskript. zgl. Habilitationsschrift Dortmund.

Gängler, H. (2010). Die Sozialpädagogik und ihre Didaktik. In D. Kasüschke (Hrsg.), Didaktik in der Pädagogik der frühen Kindheit (S. 360384). Köln \& Kronach: Link.

Gängler, H., \& Liebig, M. (2017). Lehren und Lernen in der Sozialpädagogik. In F. Kessl, E. Kruse, S. Stövesand \& W. Thole (Hrsg.), Soziale Arbeit - Kernthemen und Problemfelder (S. 253-259). Opladen: Barbara Budrich.

Göddertz, N. (2021). Sozialpädagogische Qualifizierungen weiterdenken. Sozial Extra. https://doi.org/10.1007/s12054-021-00414-7.

Göddertz, N., \& Karber, A. (2019). Berufliche Bildung Sozialpädagogik - Eine Spurensuche didaktischer Prinzipien. Soziale Passagen: Journal Für Empirie und Theorie Sozialer Arbeit, 11(1), 65-80.

Gruschka, A. (2011). Erziehen heißt Verstehen lehren. Ein Plädoyer für guten Unterricht. Stuttgart: Reclam.

Hamburger, F. (2008). Einführung in die Sozialpädagogik. Stuttgart: Kohlhammer.

Klafki, W. (1996). Neue Studien zur Bildungstheorie und Didaktik: zeitgemäße Allgemeinbildung und kritisch-konstruktive Didaktik. Weinheim \& Basel: Beltz.

KMK (2019). Ländergemeinsame inhaltliche Anforderungen für die Fachwissenschaften und Fachdidaktiken in der Lehrerbildung. https://www.kmk.org/fileadmin/Dateien/veroeffentlichungen_ beschluesse/2008/2008_10_16-Fachprofile-Lehrerbildung.pdf. Zugegriffen: 31. Jan. 2020.

Liebig, M. (2020). Arbeitsaufgabenbezug in der beruflichen Didaktik der Sozialpädagogik. Neue Ansätze für das Lehren und Lernen. Bielefeld: wbv.

Liebig, M., \& Gängler, H. (2018). Lehrerinnen- und Lehrerbildung als Persönlichkeitsbildung. http://www.bwpat.de/ausgabe34/liebig_gaengler_bwpat34.pdf. Zugegriffen: 17. Okt. 2018. bwp@ Berufs- und Wirtschaftspädagogik - online, Ausgabe 34, 1-14.

Sünker, H. (2009). Demokratie und Mündigkeit oder Disziplin und Unterwerfung. Gesellschaftliche Perspektiven von Erziehung und Bildung. In H. Sünker \& H.-U. Otto (Hrsg.), Demokratische Bildung oder Erziehung zur Unmündigkeit. Pädagogisch-politische Alternativen heute (S. 225-242). Lahnstein: neue praxis.

Thiersch, H. (1979). Lernen in der Jugendhilfe. Unsere Jugend, 31, 459467.

Thiersch, H., \& Böhnisch, L. (2014). Spiegelungen. Lebensweltorientierung und Lebensbewältigung. Gespräche zur Sozialpädagogik. Weinheim u. a.: Beltz Juventa.

Thole, W. (2012). Die Soziale Arbeit - Praxis, Theorie, Forschung und Ausbildung. Versuch einer Standortbestimmung. In W. Thole (Hrsg.), Grundriss Soziale Arbeit. Ein einführendes Handbuch (S. 19-70). Wiesbaden: Springer VS. 